Utah State University

DigitalCommons@USU

9-27-2013

\title{
Diverse electron-induced optical emissions from space observatory materials at low temperatures
}

\author{
JR Dennison \\ Utah State Univesity \\ Amberly Evans Jensen \\ Utah State University \\ Gregory Wilson \\ Utah State University \\ Justin Dekany \\ Charles W. Bowers \\ NASA Goddard Space Flight Center \\ Robert H. Meloy \\ ASRC Federal Holding Co. \\ Follow this and additional works at: https://digitalcommons.usu.edu/mp_conf \\ Part of the Physics Commons
}

\section{Recommended Citation}

JR Dennison, Amberly Evans Jensen, Justin Dekany, Gregory Wilson, Charles W. Bowers and Robert Meloy, "Diverse Electron-induced Optical Emissions from Space Observatory Materials at Low Temperatures," Proceedings of the Society of Photo-Optical Instrumentation Engineers Cryogenic Optical Systems and Instruments Conference, Vol. 8863, 2013, pp. 88630B1-88630B15. DOI: 10.1117/

12.2030232

This Article is brought to you for free and open access by the Materials Physics at DigitalCommons@USU. It has been accepted for inclusion in Conference Proceedings by an authorized administrator of DigitalCommons@USU. For more information, please contact digitalcommons@usu.edu.

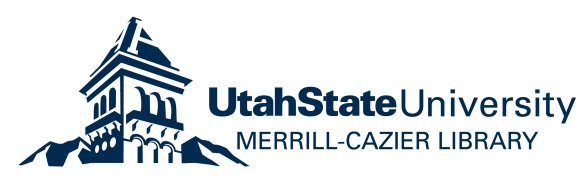




\title{
Diverse Electron-induced Optical Emissions from Space Observatory Materials at Low Temperatures
}

\author{
JR Dennison ${ }^{*}$, Amberly Evans Jensen ${ }^{\mathrm{a}}$, Gregory Wilson ${ }^{\mathrm{a}}$, Justin Dekany ${ }^{\mathrm{a}}$, \\ Charles W. Bowers ${ }^{\mathrm{b}}$, and Robert Meloy ${ }^{\mathrm{c}}$ \\ ${ }^{a}$ Materials Physics Group, Physics Department, Utah State University \\ ${ }^{\mathrm{b}}$ NASA Goddard Space Flight Center $\quad{ }^{\mathrm{c}}$ ASRC Federal AS\&D, Inc.
}

\begin{abstract}
Electron irradiation experiments have investigated the diverse electron-induced optical and electrical signatures observed in ground-based tests of various space observatory materials at low temperature. Three types of light emission were observed: (i); long-duration cathodoluminescence which persisted as long as the electron beam was on (ii) short-duration ( $<1 \mathrm{~s})$ arcing, resulting from electrostatic discharge; and (iii) intermediate-duration ( $100 \mathrm{~s})$ glow-termed "flares". We discuss how the electron currents and arcing - as well as light emission absolute intensity and frequency - depend on electron beam energy, power, and flux and the temperature and thickness of different bulk (polyimides, epoxy resins, and silica glasses) and composite dielectric materials (disordered $\mathrm{SiO}_{2}$ thin films, carbon- and fiberglass-epoxy composites, and macroscopically-conductive carbon-loaded polyimides). We conclude that electron-induced optical emissions resulting from interactions between observatory materials and the space environment electron flux can, in specific circumstances, make significant contributions to the stray light background that could possibly adversely affect the performance of space-based observatories.
\end{abstract}

Keywords: space environment, light emission, cathodoluminescence, optical coatings, cryogenic, arcing, space-based observatories

\section{INTRODUCTION}

New and proposed space-based observatories potentially subject optical elements and ancillary support structures to significant space environment electron fluxes ${ }^{1}$. An open architecture and minimal shielding are often required for such large space-based observatories, due to size and mass constraints. The open structure exposes large areas of optical elements and surrounding support structures to electron fluxes and also permits stray light to enter the optical path of the telescope ${ }^{2}$. Exposure to environmental electron fluxes can lead to enhanced accumulation of charge (particularly at low operating temperatures) ${ }^{3}$. This can also enhance the possibility that electron-induced optical emission can lead to performance degradation unless steps are taken to mitigate this risk.

The principle question addressed in this paper is: Can electron-induced optical emissions resulting from interactions of space environment electron fluxes with observatory materials make significant contributions to the stray light background that could adversely affect the performance of space-based observatories? The relative importance of electron-induced light emission for spaced-based observatories is determined by comparison of the light intensity produced by relatively low-level space plasma fluxes to both external and internal sources of background light levels ${ }^{1,4}$.

Significant contributions to the background light levels of some space-based observatories have been identified as the external celestial sky background and thermal self-emission of optical components within the observatories ${ }^{5,6}$. The celestial sky background is comprised of: (i) a contribution from sources beyond our solar system referred to as the galactic background and (ii) a contribution from sources within our solar system referred to as the zodiacal background, which includes reflected sunlight and thermal emissions from interplanetary zodiacal dust. In addition, Earth and lunar glow backgrounds result from similar reflection of sunlight reflected from the Earth and moon scattering off of zodiacal dust. Ferguson et al. discuss these background sources in near Earth orbits, and conclude that under many observation

*JR.Dennison@.usu.edu; phone 435-797-2936

Cryogenic Optical Systems and Instruments 2013, edited by James B. Heaney,

E. Todd Kvamme, Proc. of SPIE Vol. 8863, 88630B · (c) 2013 SPIE

CCC code: $0277-786 \mathrm{X} / 13 / \$ 18 \cdot$ doi: $10.1117 / 12.2030232$

Proc. of SPIE Vol. $886388630 \mathrm{~B}-1$ 
conditions the zodiacal background is the limiting external stray light component ${ }^{4}$. Likewise, Lightsey et al. have concluded that the natural background from the zodiacal dust is the dominant external stray light source for visible and NIR background for the L2 space environment further from the Earth ${ }^{2}$, where contributions from the Earth and Moon shine and from bright point sources near the field of view were shown to be at least an order of magnitude less than the in-field background of the zodiacal sky ${ }^{5,7}$. For IR observatories or sensors, minimization of internal thermal background emission often requires telescope and detector operation at low temperatures down to 10's of Kelvins. Cooling to $\$ 100$ $\mathrm{K}$ ambient operating temperatures keeps stray light contamination from total self-emission IR light levels less than the stray light from the zodiacal background, except for mid-IR wavelengths $>10 \mu \mathrm{m}^{7}$.

The background flux from the zodiacal sky originating from outside an observatory, onto an observatory detector, can provide a useful figure of merit for comparison with the background produced by electron-induced emission from components within the observatory ${ }^{2,4,6}$. The spectral radiance of the zodiacal background at $1 \mathrm{AU}$ near the south ecliptic pole is $8 \cdot 10^{-14} \mathrm{~W} \mathrm{~cm}^{-2} \mathrm{~nm}^{-1} \mathrm{sr}^{-1}$ at $500 \mathrm{~nm}, 3 \cdot 10^{-14} \mathrm{~W} \mathrm{~cm}^{-2} \mathrm{~nm}^{-1} \mathrm{sr}^{-1}$ at $1000 \mathrm{~nm}$, and $6 \cdot 10^{-15} \mathrm{~W} \mathrm{~cm}^{-2} \mathrm{~nm}^{-1} \mathrm{sr}^{-1}$ at $2000 \mathrm{~nm}$ 6 . The flux of light from distant sources such as the zodiacal background at a particular wavelength onto a detector is generally just the zodiacal radiance times the field of view times the area of the primary mirror times the overall efficiency of the telescope plus detector. We should note however, that while in-field light from distant sources can travel unobstructed along the optical path, this is not necessarily the case for light which can be emitted from structures within the observatory when exposed to incident electrons. The location and size of light emitting structures, effective baffling, and the use of low scatter optics can greatly reduce the effect of such internal sources of stray light. However, as a fiducial 'faint source', the zodiacal sky radiance, even without these other considerations, provides a useful reference value. The intensity of light emissions for a given incident electron flux are presented here as absolute spectral radiance values. In essence, this provides direct comparison of the faint background from a distance source seen by a detector to emission from an area of a distance emitting material subtending a similar solid angle in a similar wavelength range. The specific effects for each observatory must be accounted for through additional calculations of an effective spectral radiance, which incorporates the efficiency with which a specific observatory detector captures emission from a specific internal material source.

Typical space plasma environments have electron energy spectra extending from 10's of eV up to $\sim 1 \mathrm{MeV}$ with integrated fluxes of $<0.01 \mathrm{pA}-\mathrm{cm}^{-2}$ to $>10 \mathrm{nA}-\mathrm{cm}^{-2} 1,8$. Environmental electron fluxes exhibit a wide variance due to storm conditions, stochastic variability and changes due to the orbit of the observatories ${ }^{8}$. However, a reasonable estimate of incident electron power in severe space weather storm conditions for typical environments is on the order of $10 \mu \mathrm{W}-\mathrm{cm}^{-2}$ (i.e., $10 \mathrm{nA}-\mathrm{cm}^{-2}$ and $1 \mathrm{keV}$ or $1 \mathrm{nA}-\mathrm{cm}^{-2}$ and $10 \mathrm{keV}$ ); this provides a scaling factor for comparison of electron-induced optical emissions observed in the experiments described below to realistic space conditions. More realistic calculations can be made using an average indecent electron power appropriate for a specific observatory; even better is to calculate emissions based on integration over a differential electron flux as a function of incident power ${ }^{1,12-14}$.

We review laboratory electron irradiation experiments we conducted to investigate the diverse electron-induced optical and electrical signatures observed in tests of several space observatory materials at low temperature. Various experiments measured electron-induced absolute photon emission yields and photon emission spectra, arcing rates and location, transport and displacement currents to a rear grounded electrode, and absolute electron emission yields. Numerous arcing events were observed (particularly at lower temperatures), consistent with enhanced charge accumulation due to lower conductivities at low temperature. Three types of light emission were also observed: (i) shortduration $(<1 \mathrm{~s})$ arcing, resulting from electrostatic discharge; (ii) long-duration continuous emission present as long as the electron beam was on, called cathodoluminescence; and (iii) intermediate-duration ( 100 s) glow-termed "flares"that dissipated exponentially with time after infrequent and rapid onset. We discuss how the electron currents and arcing - as well as light emission absolute intensity and frequency - depend on electron beam energy and flux, deposited power, and material temperature and thickness. The different bulk and composite insulating materials studied provide examples of thin, thick, and multi-thickness dielectrics that can be modeled with the same simple approach to predict the dependence of light emission that might be encountered in space applications.

\section{EXPERIMENTATION}

Experiments measured the electrical and optical response of sample materials bombarded with a beam of electrons. Experiments were conducted in the main Utah State University electron emission ultrahigh vacuum test chamber ${ }^{10}$, which had been modified for observations of low intensity UV/Vis/NIR glow over a broad range of sample temperatures ${ }^{11}$. Figure 1 provides a schematic of the experimental system used. Measurements were conducted from $<40-290 \mathrm{~K}$, using well-characterized, low-flux electron beams to deposit electrons near the sample surfaces. Details of 


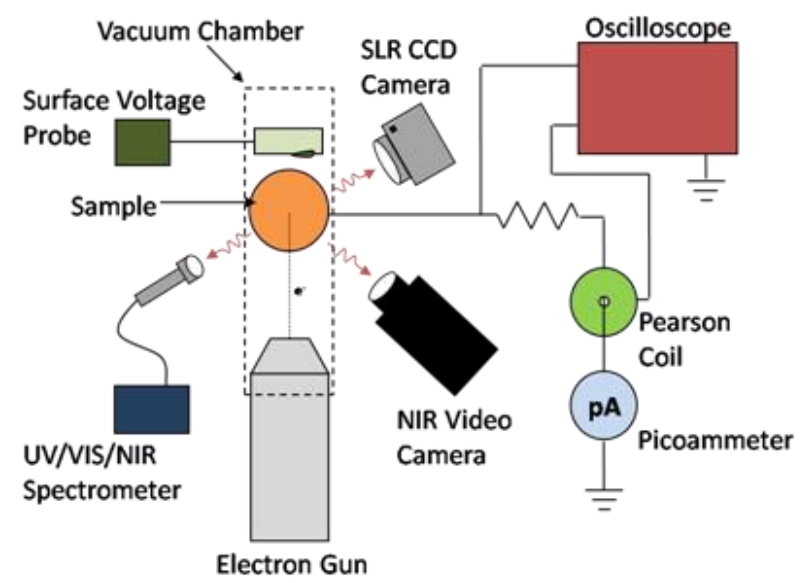

Figure 1. (a) Block diagram of instrumentation for collecting the pulse charging surface voltage, electrode current and cathodoluminescence data induced by electron beam bombardment. Instrumentation includes picoammeters, Pearson coils, and a storage oscilloscope for electrode current measurements and UV/Vis and IR spectrometers, an SLR CCD still camera, a CCD video camera, and a NIR video camera for optical measurements.

the experimental set up; electron sources and beam characterization; optical detectors, methods, and calibration; the sample mounting carousel and thermal stage; and a general schematic of the experimental system used are given by Dennison et al. ${ }^{10}$. Additional details of the thermal dependence of the cathodoluminescence are presented elsewhere ${ }^{1,12-}$ ${ }^{14}$. Electron transport, surface voltage data, and electrostatic discharge measurements acquired simultaneously with luminescence data are also reported elsewhere ${ }^{12}$.

Electrons beams with $\sim 50 \mathrm{pA}-\mathrm{cm}^{-2}$ to $1 \mu \mathrm{A}-\mathrm{cm}^{-2}$ current densities and $0.2 \mathrm{keV}$ to $30 \mathrm{keV}$ energies were directed on to the samples. The defocused beam profiles at the sample were uniform to about $\pm 30 \%$ over an $\sim 3 \mathrm{~cm}$ diameter beam. Beam fluxes were measured before and after each experiment with a Faraday cup and were stable to within approximately $\pm 5 \%$. The filaments of the electron gun also produced light that was visible on the sample. A small bright spot near the center of the sample from the blackbody radiation of the $\sim 1700 \mathrm{~K} \mathrm{LaB}_{6}$ filament of one of the electron guns is visible in many of the images shown in Figs. 2, 3, 4 and 7; this spot provides a roughly constant $20 \mathrm{pW}-\mathrm{cm}^{-2}$ signature that is useful for comparison. For the brighter W filament $(\sim 2500 \mathrm{~K})$ used in another electron gun, the intensity of the filament spot on the sample was larger than the observed luminescent signals. To minimize this contaminating filament light, a blocking aperture was developed, which reduced the stray light contamination seen by the optical cameras to acceptable levels ${ }^{3}$.

Two cameras and two fiber optic spectrometers were used to monitor low light intensity. Optical data were collected using UV/Vis and NIR spectrometers, an SLR CCD still camera, a Vis/NIR image-intensified CCD video camera, an InGaAs NIR video camera, and a mid-IR InSb video camera. For each optical detector, the absolute spectral response and wavelength range were determined and the sensitivity was calibrated with NIST traceable sources prior to the luminescence studies ${ }^{11,13}$. Specifications and sensitivities of the optical and electron detectors used for the studies reported here are summarized in Table 1.

A wide variety of thin film dielectric and nanodielectric composite samples were tested for the studies described here. These included polyimide films, neat urethane and bisphenol/amine epoxy films, thin optical coatings of disordered $\mathrm{SiO}_{2}{ }^{1,12-14}$, several grades of commercially available high-conductivity carbon-loaded polyimide nanodielectric composites ${ }^{13,14}$, cyanate ester and urethane epoxy resins in graphite fiber and fiberglass composites ${ }^{14}$, and multilayer dielectric/conductor composites ${ }^{3}$. The $10 \mathrm{~mm}$ to $25 \mathrm{~mm}$ diameter samples tested for these studies were optically cleaned and underwent a $\sim 12 \mathrm{hr}$ vacuum bakeout at $\sim 390 \mathrm{~K}$ and $<1 \bullet 10^{-3} \mathrm{~Pa}$ to eliminate adsorbed water and volatile contaminates. The samples were placed in an ultrahigh vacuum chamber (base pressure $<1 \bullet 10^{-6} \mathrm{~Pa}$ ) for $>24 \mathrm{hrs}$ to allow for outgassing before measurements were made. The samples were mounted on Cu pedestals with an $\sim 0.5 \mathrm{~mm}$ to $1.5 \mathrm{~mm}$ gap between the sample and a grounded sample carousel; most samples were mounted flush with the top of the sample carousel. The sample carousels were thermally anchored to (but electrically isolated from) a thermal reservoir. In some experiments, using a combination with resistive heaters and liquid $\mathrm{N}_{2}$ cryogen, the samples were maintained over a range of temperatures from $\sim 150 \mathrm{~K}$ to $\sim 400 \mathrm{~K}$ with a stability of $\pm 4 \mathrm{~K}$ over typical $2 \mathrm{hr}$ experiment 

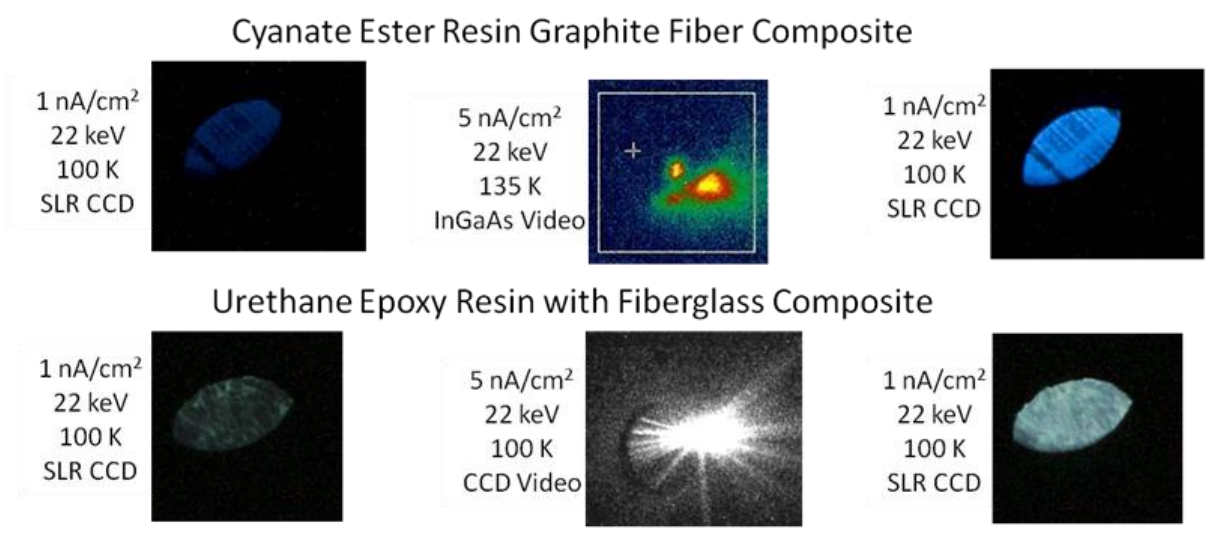

\section{Disordered $\mathrm{SiO}_{2}$ Coating}
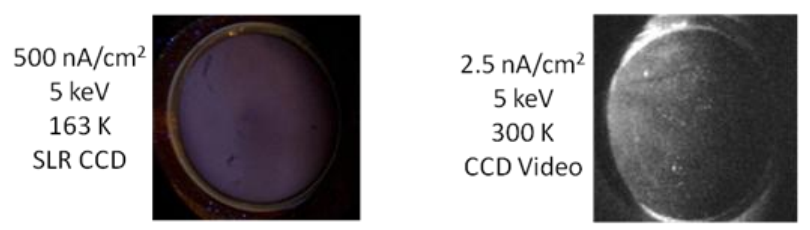

Not seen for

this material

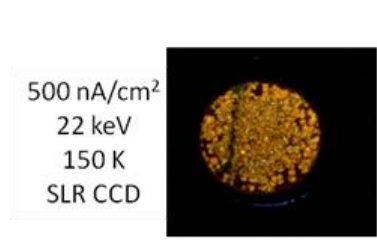

Kapton E Bulk Polyimide

High Conductivity Carbon Loaded Polyimide

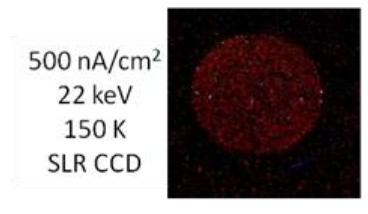

Sustained Glow
Not seen for

this material
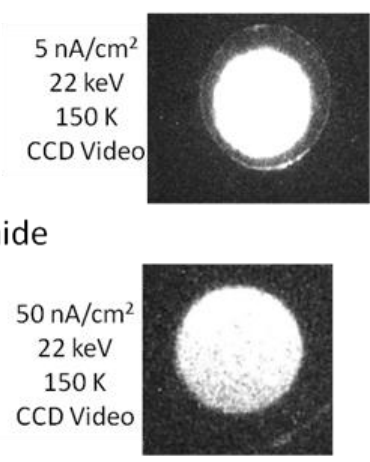

"Flares"

Figure 2. Representative images of electron-induced optical emissions from dielectric materials. Images are shown for five different materials and for sustained glow, short-duration arcs, and intermediate-duration "flares". Samples are $10 \mathrm{~mm}$ to $25 \mathrm{~mm}$ in diameter, mounted with a $0.5 \mathrm{~mm}$ to $1.5 \mathrm{~mm}$ gap between the samples and grounded conducting sample holders. Images were taken using one of three cameras as noted in the captions: an SLR CCD camera at 30 s/frame with RGB images over $390 \mathrm{~nm}$ to $650 \mathrm{~nm}$; an image-intensified CCD video camera at $30 \mathrm{frames} / \mathrm{s}$ over $400 \mathrm{~nm}$ to $900 \mathrm{~nm}$; and an InGaAs video camera at 60 frames/s over $950 \mathrm{~nm}$ to $1700 \mathrm{~nm}$ (see Table 1 for instrumentation details.). The incident electron current density and energy and the sample temperature for each image are also noted in the captions.

durations. In other experiments from $<40 \mathrm{~K}$ to $\sim 350 \mathrm{~K}$, a closed cycle He refrigerator system with a stability of $\pm 0.5 \mathrm{~K}$ was used ${ }^{11}$. Wires were attached to the samples to collect electrical data during bombardment as well; these results are reported elsewhere ${ }^{3}$.

\section{OPTICAL EMISSIONS}

All types of samples studied here exhibited readily observable electrical discharges and luminescence when subjected to electron beam bombardment, as illustrated in Figs 2 and 3. Three types of light emission with simultaneous current signatures were observed: (i) long-duration sustained glow (cathodoluminescence), which persisted as long as the electron beam was on; (ii) short-duration $(<1 \mathrm{~s})$ arcing, resulting from electrostatic discharge; and (iii) intermediate duration $\left(\sim 10^{1}-10^{2} \mathrm{~s}\right)$ emissions termed "flares", that dissipated exponentially with time after infrequent and rapid onset. 
Table 1. Detector Specifications and Sensitivity

\begin{tabular}{|c|c|c|c|c|c|}
\hline Sensor & $\begin{array}{l}\text { Bandpass / } \\
\text { Bandwidth } \\
\quad(\mathrm{nm})\end{array}$ & $\begin{array}{c}\text { Weighted } \\
\text { Central } \\
\text { Wavelength } \\
\text { (nm) }\end{array}$ & Data Rate & $\underset{\mathrm{a}}{\operatorname{Resolution}}$ & $\begin{array}{c}\text { Detection } \\
\text { Threshold Spectral } \\
\text { Radiance }^{\mathrm{b}} \\
{\left[\mathrm{W}-(\mathrm{cm}-\mathrm{nm}-\mathrm{sr})^{-1}\right]}\end{array}$ \\
\hline $\begin{array}{l}\text { SLR CCD Camera } \\
\text { (Cannon, EOS Rebel XT } \\
\text { DS126071) }\end{array}$ & $\begin{array}{l}390-650 \\
260\end{array}$ & 553 & $30 \mathrm{~s} /$ frame & $\begin{array}{c}\sim 5 \\
\mu \mathrm{m} / \mathrm{pixel}\end{array}$ & $4 \cdot 10^{-15}$ \\
\hline $\begin{array}{l}\text { Image-intensified CCD Video } \\
\text { Camera (Xybion, ISG-780-U-3) }\end{array}$ & $\begin{array}{c}400-900 \\
500\end{array}$ & 830 & 30 frames $/ \mathrm{s}$ & $\begin{array}{c}\sim 30 \\
\mu \mathrm{m} / \text { pixel }\end{array}$ & $4 \cdot 10^{-16}$ \\
\hline $\begin{array}{l}\text { InGaAs Video Camera } \\
\text { (Goodrich, SU320MS-1.7RT) }\end{array}$ & $\begin{array}{l}950-1700 \\
750\end{array}$ & 1512 & 60 frames/s & $\begin{array}{c}\sim 30 \\
\mu \mathrm{m} / \text { pixel }\end{array}$ & $8 \cdot 10^{-16}$ \\
\hline $\begin{array}{l}\text { InSb Video Camera } \\
\text { (Santa Barbara Focalplane, SBF119) }\end{array}$ & $\begin{array}{c}1000-5500 \\
4500\end{array}$ & 3900 & 15 frames/s & $\begin{array}{c}\sim 100 \\
\mu \mathrm{m} / \text { pixel }\end{array}$ & $\sim 2 \cdot 10^{-11}$ \\
\hline $\begin{array}{l}\text { UV/Vis Spectrometer } \\
\text { (Stellarnet, 13LK-C-SR) }\end{array}$ & $200-1080$ & NA & $\sim 10 \mathrm{scans} / \mathrm{s}$ & $<0.5 \mathrm{~nm}$ & $\sim 2 \cdot 10^{-11}$ \\
\hline $\begin{array}{l}\text { NIR Spectrometer } \\
\text { (Stellarnet, RW-InGaAs-512) }\end{array}$ & $1000-1700$ & NA & $\sim 10 \mathrm{scans} / \mathrm{s}$ & $<2 \mathrm{~nm}$ & $\sim 1 \cdot 10^{-11}$ \\
\hline Electrometer (Custom Design ${ }^{9}$ ) & $\mathrm{NA}$ & NA & $\sim 30 \mathrm{~ms} /$ point & $<0.2 \mathrm{pA}$ & NA \\
\hline $\begin{array}{l}\text { Digital Storage Oscilloscope } \\
\text { (Tektronix TDS 2100) }\end{array}$ & NA & NA & $\begin{array}{c}20 \mu \mathrm{s} / \mathrm{scan} \\
0.1 \mathrm{~ns} / \text { point }\end{array}$ & $<200 \mathrm{pA}$ & NA \\
\hline
\end{tabular}

Sustained glow, arcs, and flares were all detected in the electrometer, oscilloscope, Vis SLR camera, Vis/NIR CCD video camera, and NIR InGaAs video camera; coincidence was almost always seen, except when the signals were below detection thresholds for specific instruments.

\subsection{Long-duration Cathodoluminescence}

Electron-induced glow, or cathodoluminescence, was observed when $\mathrm{keV}$ energy electrons bombard certain dielectric surfaces over a wide range of temperatures. Previous studies have observed similar cathodoluminescence in both epoxy resin composites ${ }^{15}$ and in disordered $\mathrm{SiO}_{2}$ films ${ }^{16-19}$ at room temperature; however, little information was available with regard to the temperature dependence of the cathodoluminescence or the absolute magnitude of the emissions. The cathodoluminescence turned on and off with the incident electron beam (with response times of $\lesssim 1 \mathrm{~s}$ ) and was visible over the full illuminated sample area, as seen in Figs. 2 and 4. The optical emission intensity scaled with deposited electron beam power (see below) and responded to changes in electron beam position and profile on rapid time scales ${ }^{1}$. At higher incident power levels, equilibrium glow intensity was sometimes seen to decrease $\sim 2 \mathrm{X}$ due to long exposure of beam, as the accumulated negative surface charge or reduced landing energy increased the electron yield, $\sigma_{\text {yield }}\left(E_{b}, \Sigma\right)$, toward unity. The incident beam energy is $E_{b}$.

Figure 3 shows that there is excellent temporal correlation between the electrometer data and the video camera spectral radiance curves. Sustained glow intensity or current takes a finite amount of time to reach a fairly steady equilibrium value. There is also a finite decay time seen in glow intensity and current curves after the beam is turned off (see Fig. 3). These exponential rise and decay time constants $\left(\sim 10^{-1} \mathrm{~s}\right)$ are roughly the same and are believed to be related to filling and release rates of electrons in the trap states of the insulators. Negative currents result from displacement currents to the grounded rear sample electrode and the grounded sample holder, as negative charge from the electron beam accumulates in the trap states of the sample. The magnitude of the sample currents during beam-on times was consistent with a displacement current that resulted as charge accumulated in the sample. The measured rate of surface charging $d \Sigma / d t$ was typically close to the incident beam current density, $J_{b}$, reduced by the total electron yield of the sample, i.e., $d \Sigma / d t=J_{b}\left[1-\sigma_{\text {yield }}\left(E_{b}, \Sigma\right)\right]$.

A more quantitative measure of the cathodoluminescent intensity was obtained from the signal of Vis/NIR CCD and InGaAs NIR video cameras averaged over the illuminated area of the sample, as shown in Figs. 3(b-c). These plots are given in terms of calibrated absolute spectral radiance for the beam currents, beam energies, and temperatures noted. Simple models propose that luminescence increases linearly with incident power density (beam energy times beam 


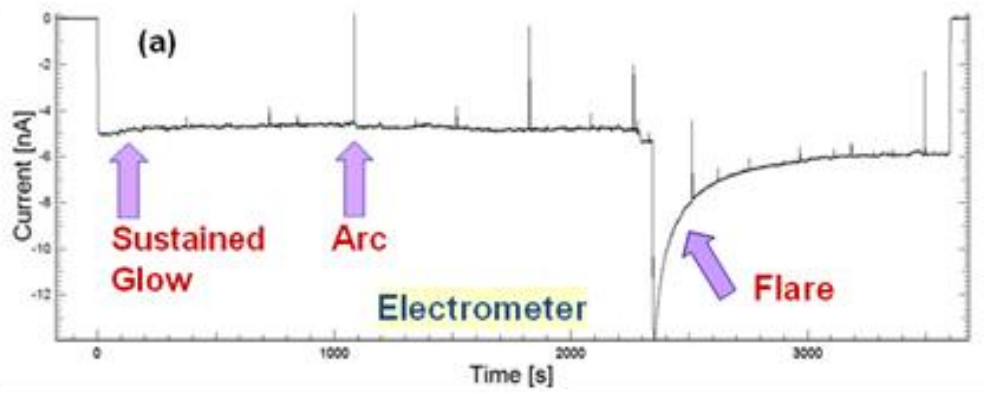

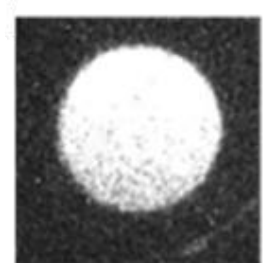

A

(d)

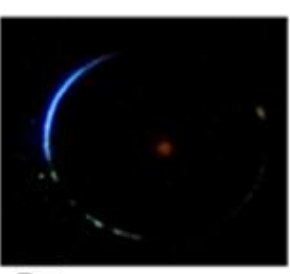

B

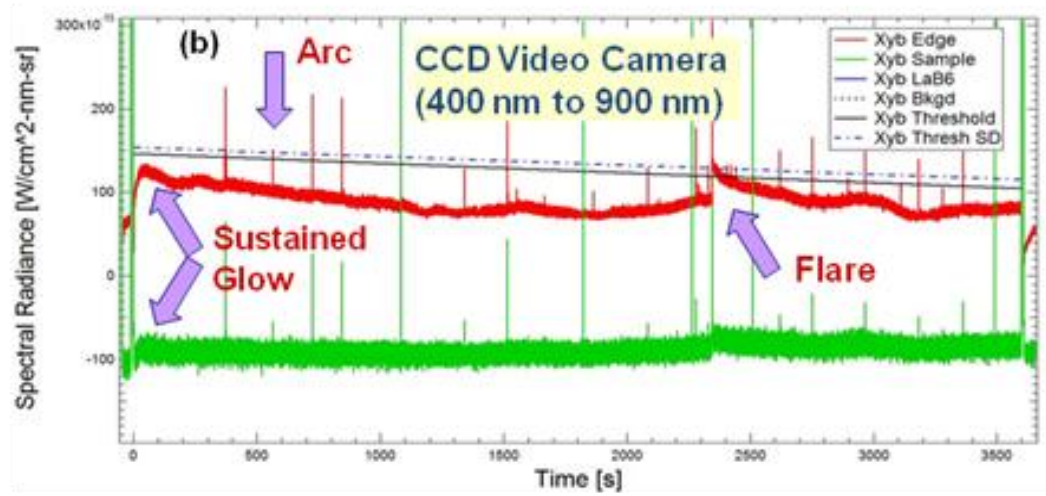

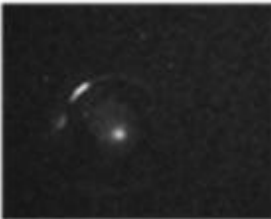

1

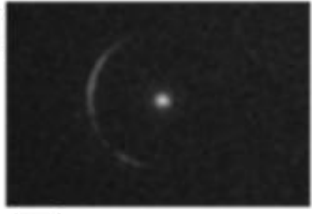

1

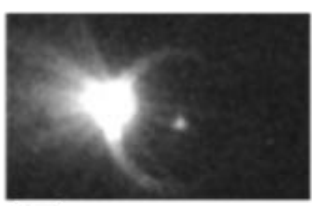

3

(e)

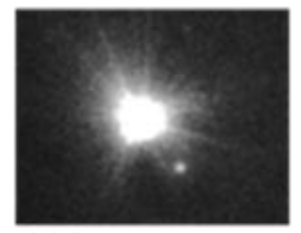

2

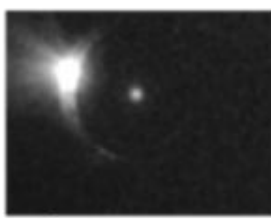

2

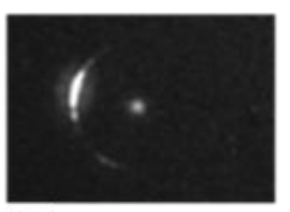

4

(f)

Figure 3. (a-c) Intensity profiles of sample under electron bombardment. The carbon-loaded polyimide sample is $1 \mathrm{~cm}$ in diameter and measured at $\sim 110 \mu \mathrm{W}-\mathrm{cm}^{-2}$ electron power density $\left(5 \mathrm{nA}-\mathrm{cm}^{-2}\right.$ at $\left.22 \mathrm{keV}\right)$ at $135 \mathrm{~K}$. (a) Electrometer sample current as a function of elapsed time. A nearly constant current of $\sim 5 \mathrm{nA}$ associated with the sustained glow is observed from $0 \mathrm{~s}$ to $3600 \mathrm{~s}$, while the beam is on. Large short-duration arcs are observed at $\sim 1050 \mathrm{~s}, \sim 1820 \mathrm{~s}, \sim 2280 \mathrm{~s}, \sim 2490 \mathrm{~s}$ and $\sim 2480 \mathrm{~s}$; numerous smaller arcs are also evident. A large abrupt current spike at $\sim 2345 \mathrm{~s}$ - with an approximately exponential decay with a $\sim 50$ s decay time-is labeled a "flare". (b) Absolute spectral radiance as a function of elapsed time measured with the CCD video camera. The green and red curves are the average intensities for the central sample surface and edge (region near the sample-sample holder gap) regions. Note the spectral radiance in (b) and (c) due to glow is measures as the change from the beam-off intensities at the beginning and end of the curves. (c) Absolute spectral radiance as a function of elapsed time measured with the InGaAs video camera. Note only the larger arcs seen in the electrometer and CCD video camera profiles are evident in the InGaAs profile. (d-f) CCD video images at 30 frames/s of the same sample. A small bright spot from the blackbody radiation of the $\sim 1700 \mathrm{~K}$ electron gun $\mathrm{LaB}_{6}$ filament is evident near the center of most images. (d) A-Image of surface cathodoluminescence extending over the full surface at $\sim 50 \mathrm{nA}$ $\mathrm{cm}^{-2}$, B-SLR CCD $30 \mathrm{~s}$ exposure showing edge glow taken at $\sim 5 \mathrm{nA}-\mathrm{cm}^{-2}$. (e) Successive frames before and just after the onset of a "flare" event. Subsequent frames are similar to frame 2 showing emission over the full sample surface, with gradually decreasing intensity as shown in Fig. 9. (f) Successive frames before, during and after an arc event at the edge. 


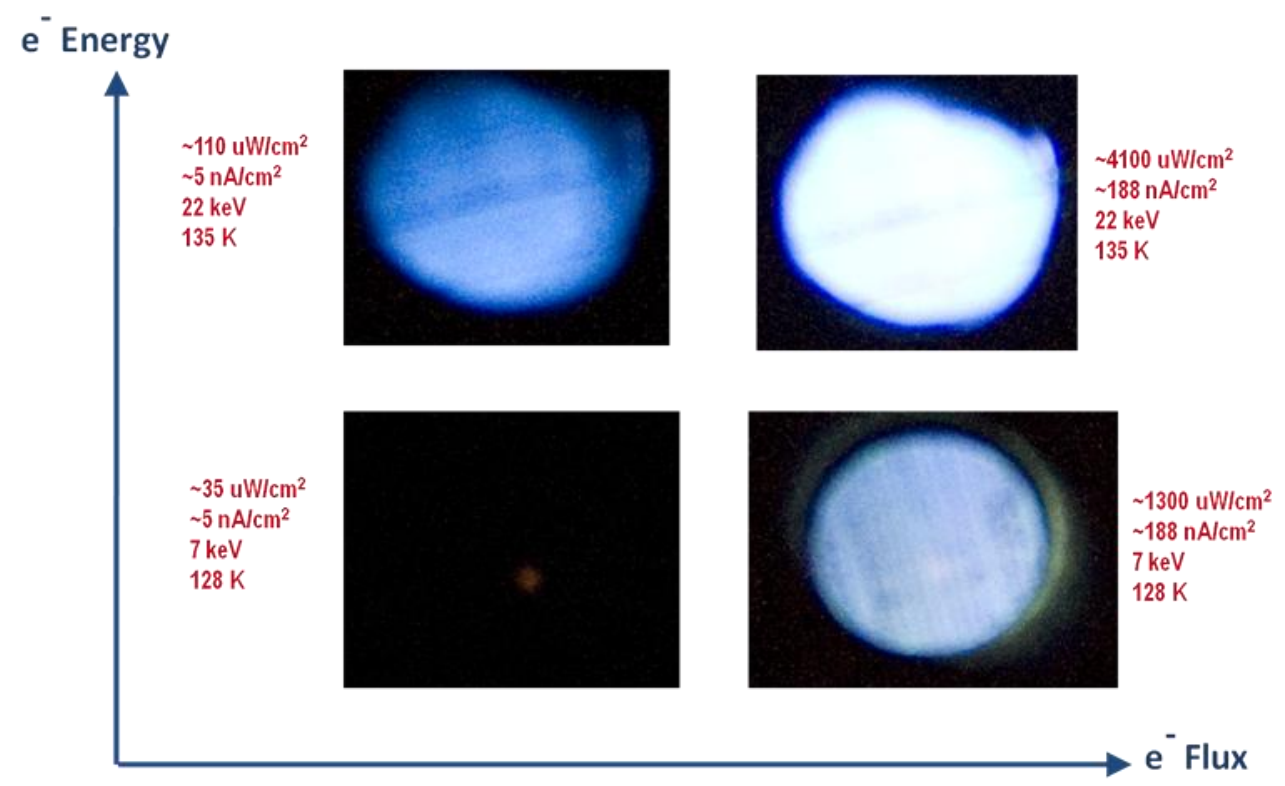

Figure 4. Cathodoluminescence of cyanate ester graphite fiber composite material. The four images were taken at the electron energies, current densities, and power densities noted in the captions at approximately the same temperature. Intensity is seen to increase with increasing electron energy and flux. Note the structure in the images resulting from the graphite fiber structure and varying thickness of the epoxy resin

current density) for non-penetrating radiation. For many materials, total luminescent radiance increased with increasing incident beam current and energies at fixed temperature, as seen in Fig. 4. More correctly, the luminescence increases with deposited power. This explains the complex behavior of the spectral radiance curves with increasing energy seen in Fig. 5. For the non-penetrating behavior of a bulk cyanate ester epoxy composite material, the spectral radiance increased with increasing electron energy (Note, this curve exhibits saturation effects at higher energies.) In contrast, the spectral radiance decreased with increasing energy for the penetrating behavior of a disordered $\mathrm{SiO}_{2}$ thin film. The spectral radiance of a high-conductivity carbon-loaded polyimide nanodielectric composite ${ }^{13,14}$ sample was largely independent of absorbed power; it has been proposed that a combination of thick polyimide regions (with nonpenetrating radiation) and thin polyimide layers coating near-surface carbon particles (with penetrating radiation) can produce such a signature nearly independent of incident power ${ }^{13}$.

Measurements for many different materials have determined approximate cathodoluminescent intensities for keV electron bombardment at $10 \mu \mathrm{W}-\mathrm{cm}^{-2}$, representative of severe space environments. The most intense emissions were seen for epoxy materials: when compared to the zodiacal background in the visible range bulk urethane epoxy was $\sim 50 \mathrm{X}$ more intense, neat bisphenol/amine epoxy films were $\sim 20 \mathrm{X}$ more intense, cyanate ester/ graphite fiber composites were $\sim 20 \mathrm{X}$ more intense, epoxy/fiberglass composites were $\sim 5 \mathrm{X}$ more intense, and urethane epoxy/carbon fiber composites were $\sim 4 \mathrm{X}$ more intense ${ }^{13}$. Disordered $\mathrm{SiO}_{2}$ optical coating emissions were about the same intensity as the zodiacal background ${ }^{1,12-14}$. Bulk polyimide samples were $\sim 0.05 \mathrm{X}$ as intense as the zodiacal background. High-conductivity carbon-loaded polyimide samples were $\sim 0.5 \mathrm{X}$ to $\sim 0.1 \mathrm{X}$ as intense as the zodiacal background; lower intensities were observed for higher conductivity types of carbon-loaded polyimide ${ }^{12,13}$.

Overall total luminescent radiance in the visible range at fixed incident energy and incident flux increased as temperatures decreased. Observations showed about an order of magnitude increase in glow from room temperature down to $\sim 100 \mathrm{~K}$ for disordered $\mathrm{SiO}_{2}{ }^{1,12}$ and carbon-loaded polyimide ${ }^{13}$ materials. A clear change in the intensity and color was seen with the SLR images of disordered $\mathrm{SiO}_{2}$ films ${ }^{1,12}$; as temperature increased, the light emission from the samples became bluer and less red.

Jensen et al have proposed a general model of cathodoluminescent intensity based on band theory of highly disordered insulating materials that largely explains the quantitative data outlined above ${ }^{1,12}$. A more detailed discussion of the model is given elsewhere ${ }^{1,12}$. The observed luminescence occurs when an incident high energy, charged particle excites 


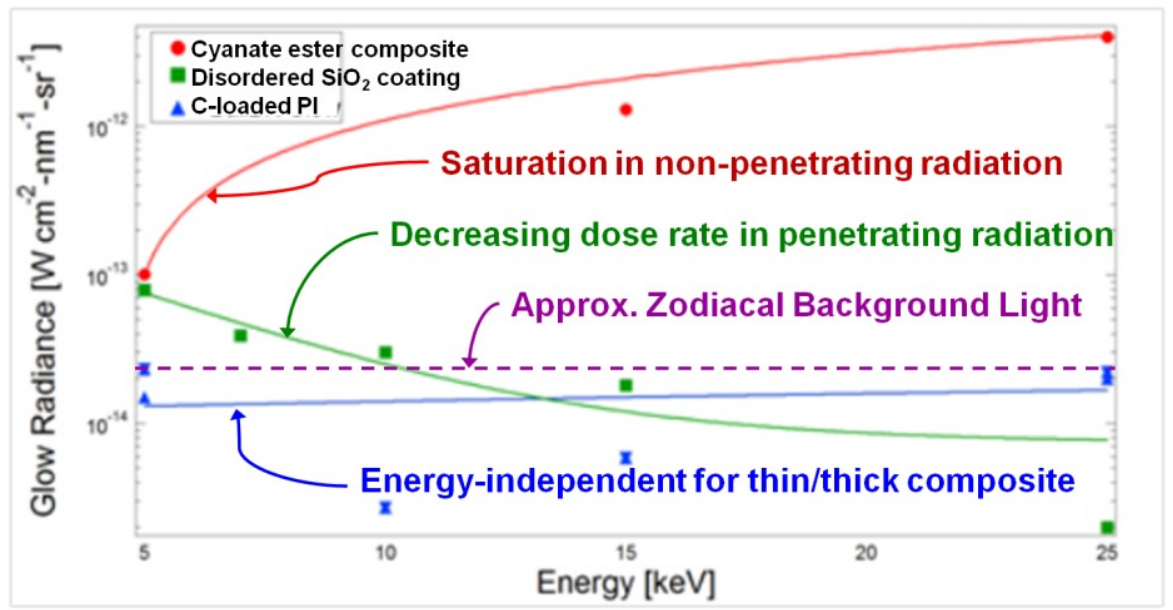

Figure 5. Cathodoluminescence spectral radiance versus incident electron energy for three materials at $10 \mathrm{nA}-\mathrm{cm}^{-2}$ electron power density, taken with the CCD video camera. The approximate level of the zodiacal background is shown for comparison.

a valence band electron into the conduction band. Thus, the photoemission power, $P_{\gamma}$, scales with incident electron current density $J_{b}$, incident electron energy $E_{b}$, and temperature $T$, as

$$
P_{\gamma}\left(J_{b}, E_{b}, T, \lambda\right) \propto\left[\frac{\dot{D}\left(J_{b}, E_{b}\right)}{\dot{D}+\dot{D}_{S a t}}\right]\left\{\left[e^{-\left(\varepsilon_{S T} / k_{B} T\right)}\right]\left[1-e^{-\left(\varepsilon_{S T} / k_{B} T\right)}\right]\right\}
$$

$P_{\gamma}$ is proportional to the number of electrons excited by the incident radiation through the dose rate $\dot{D}$ (absorbed power per unit mass). $\dot{D}$ is given as the number areal density of incident electrons $\left(J_{b} / q_{e}\right)$ times energy per incident electron $E_{b}$ divided by the mass into which energy is deposited $\left(\rho_{m} L \cdot\right.$ Area $)$ :

$$
\dot{D}\left(J_{b}, E_{b}\right)=\frac{E_{b} J_{b}\left[1-\eta\left(E_{b}\right)\right]}{q_{e} \rho_{m}} \times\left\{\begin{array}{cc}
{[1 / L]} & ; R\left(E_{b}\right)<L \\
{\left[1 / R\left(E_{b}\right)\right]} & ; R\left(E_{b}\right)>L
\end{array}\right.
$$

An energy-dependent correction to the incident flux, $J_{b}\left[1-\eta\left(E_{b}\right)\right]$, is included in Eq. (2) to account for quasi-elastic backscattered electrons that do not deposit substantial energy. For biased samples, or when excess charge is stored in the trap states, a surface voltage $V_{s}$ results and $E_{b}$ is replaced everywhere in Eqs. (1) and (2) by the landing energy, $\left(E_{b}-q_{e} V_{s}\right)$. Thus, glow intensity may diminish appreciably for highly charged surfaces. For nonpenetrating radiation - where the energy-dependent penetration depth or range $R\left(E_{b}\right)$ is less than the film thickness $L$-all incident power is absorbed in the material layer and $P_{\gamma}$ and $\dot{D}$ are linearly proportional to the incident power density, $\left(J_{b} E_{b} / q_{e}\right)$. For penetrating radiation-where $R\left(E_{b}\right)>L$-the absorbed power is reduced by a factor of $\left[L / R\left(E_{b}\right)\right]^{20}$. We assume non-penetrating radiation and incident power well below the saturation range for the calculations considered here, where luminescent intensity increases with increasing incident electron power.

The excited conduction band electron rapidly decays to localized (shallow trapped) states below the mobility edge. A final electron transition, from the short-lived shallow trap states to longer-lived deep trap states is the origin of the emitted photon. Thus, $P_{\gamma}$ in Eq. (1) is also proportional to the density of available trap states and to their trapping and retention rates. At very high current density, saturation can occur when trap states fill, limiting the number of states electrons can decay into, and leading to $P_{\gamma} \propto\left(\dot{D}+\dot{D}_{s a t}\right)^{-1}$. $\dot{D}_{\text {sat }}$ is a material dependant saturation dose rate. This saturation behavior is evident the cyanate ester composite curve shown in Fig. 5. The dose rates expected in most the space environments are small enough that saturation effects will not be evident..

The thermal dependence of luminescence in equilibrium is proportional to the number of electrons in the conduction band that can fall into the shallow traps $\left(\propto\left[e^{-\left(\varepsilon_{S T} / k_{B} T\right)}\right]\right)$ and to the fraction of electrons that are retained in the shallow 
traps and not thermally excited back into the conduction band leading to radiation induced conductivity ${ }^{21},(\propto$ $\left.\left[1-e^{-\left(\varepsilon_{S T} / k_{B} T\right)}\right]\right)$. Thus, $I_{\gamma} \propto\left\{\left[e^{-\left(\varepsilon_{S T} / k_{B} T\right)}\right]\left[1-e^{-\left(\varepsilon_{S T} / k_{B} T\right)}\right]\right\}$. At higher temperatures, where $k_{B} T \gg \varepsilon_{S T}$, the thermal energy exceeds the mean energy depth of the shallow traps below the conduction band, $\varepsilon_{S T}$, and $P_{\gamma} \propto\left(\varepsilon_{S T} / k_{B} T\right)$.

Many common spacecraft and luminescent sensor materials discussed here have broad—but distinct—visible and NIR photon emission spectra characteristic of the material ${ }^{1,12-14}$. Similar signatures have been observed in other experiments at room temperature for dielectric materials including cyanate ester and epoxy composites ${ }^{1,14,15}$, polyimide, and disordered $\mathrm{SiO}_{2}$ films ${ }^{13,16-19}$. The existence of multiple peaks in the spectra and their complex changes in peak position and intensity with temperature require extensions of the model to include multiple deep trap bands; this has been used to qualitatively explain the observed temperature dependence of spectra of disordered $\mathrm{SiO}_{2}$ coatings down to $40 \mathrm{~K}^{1,12}$

\subsection{Short-duration Arcing}

Dielectrics exposed to electron fluxes exhibited numerous short-duration $(<1 \mathrm{~s})$ optical emissions due to electrostatic discharge or arcing events, as shown in Fig. 2. In almost every case, both high speed electrical and optical signatures were evident at the same times, as shown in Figure 3.

Figures 6(a-f) show typical arc intensity curves as a function of time for oscilloscope, electrometer, CCD video camera, and InGaAs video camera measurements from small discharge areas of $\lesssim 1 \mathrm{~mm}^{2}$ (occasionally up to the full sample area of $\sim 1 \mathrm{~cm}^{2}$ ). The arc profiles typically have very rapid rise times, followed by an approximately exponential decay ${ }^{22}$. Decay time constants observed for many individual arcs and from many different materials are fairly consistent for each individual instrument, but vary substantially from instrument to instrument (oscilloscope: $10^{-7} \mathrm{~s}$ to $10^{-5} \mathrm{~s}$; electrometer: $10^{-2} \mathrm{~s}$ to $10^{0} \mathrm{~s}$; CCD camera: $10^{-2} \mathrm{~s}$ to $10^{-1} \mathrm{~s}$; InGaAs camera: $10^{-2} \mathrm{~s}$ to $10^{-1} \mathrm{~s}$ ). In general, the oscilloscope data exhibited much faster response, with nanosecond rise times and widths as short as a fraction of a microsecond ${ }^{22}$. The longer time constants exhibited in the other instruments are most likely the result of instrumental broadening and sample capacitance. Acquisition of continuous oscilloscope data at nanosecond intervals over hour-long experiments however is prohibitive, requiring that one establish trigger thresholds a priori or take data using slower detectors. These response times longer than the arc duration cause the slower instruments to record a signal averaged over the slower response times; the different response times for different instruments (see Table 1) makes cross-comparison of absolute peak amplitudes and power in the arc discharge curves from different instruments difficult. However, for most of the lower amplitude arcs, estimates of electrical power dissipated by arcs measured simultaneously with oscilloscope and electrometer agree within an order of magnitude.

Data presented in this article were acquired for $1 \mathrm{~cm}$ diameter samples held in grounded metal sample holders with 0.5 $\mathrm{mm}$ to $1.5 \mathrm{~mm}$ gaps between the sample and holder. Many of the arcs were confirmed in images to be across these gaps; see for example Figs. 2, 3 and 7. Analysis of individual frames of camera data (see Figs. 4 and 7) allowed determination of the location of each arc, with a spatial resolution of $<100 \mu \mathrm{m}$, as (almost exclusively) in the high electric field region in the gap between the sample edge and adjacent electrically isolated grounded sample holder. Often "hot spots" were observed where repeated arcs occurred, presumably as a result of higher local electric fields due to sample imperfections or asperities. The time evolution of the average intensity in individual frames of camera data (after background subtraction from dark regions) was used to monitor behavior for three separate regions (sample surface, edge, and a weak glow from the light emanating from the $\mathrm{LaB}_{6}$ filament of the electron gun at $\sim 1700 \mathrm{~K}$; refer to Fig. 4.). These intensity curves were larger for the edge regions, confirming the spatial locations of the arcs.

Surface arcs away from sample edges were seen where the separations between dielectric and conducting regions were less than the gap distance such as in: (i) thin film dielectric samples (e.g., disordered $\mathrm{SiO}_{2}$ coatings in Fig. $2^{1,12}$; (ii) thin conducting layers (e.g., $\mathrm{SiO}_{2} /$ conductor multilayers ${ }^{3}$ and floating conducting Au layers on epoxy composites in Figs. 2 and 7); and nanodielectric materials (e.g., carbon-loaded polyimide in Fig. 2, 3 and $7^{13}$ and carbon-fiber composite materials in Fig. 2). In some instances, such as shown in Fig. 7 of a composite material with an ungrounded $0.1 \mu \mathrm{m}$ $\mathrm{Au} / \mathrm{Cr}$ coating on an epoxy resin fiberglass and carbon fiber composite substrate, comparison of optical microscope images of the sample surface before and after electron bombardment showed damage sites at locations of visible arc events. Numerous damage sites from arcing are evident on the exposed sample, including the $\sim 250 \mu \mathrm{m}$ diameter features identified with the arrows. For this specific system, up to $2 \%$ of the area of the surface coating was ejected in $1 \mathrm{hr}$ of electron beam exposure. 

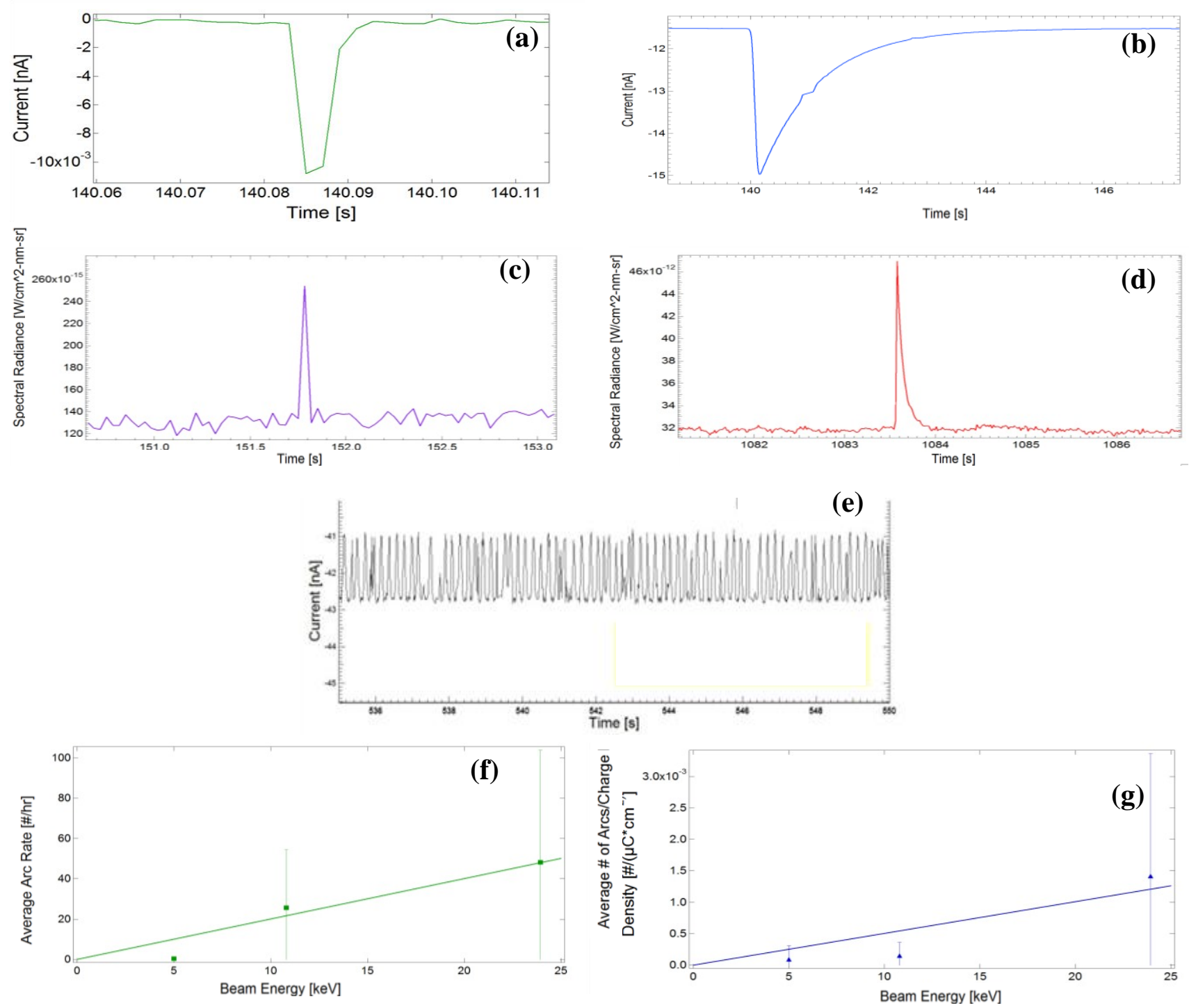

Fig. 6. Typical arc events from high-conductivity carbon-loaded polyimide samples: (a) Current versus elapsed time from oscilloscope; (b) Current versus elapsed time from electrometer; (c) Absolute spectral radiance versus elapsed time from CCD visible-range video camera; and (d) Absolute spectral radiance versus elapsed time from InGaAs NIR-range video camera. (e) Rapid arcing with $\sim 20000 \mathrm{arcs} / \mathrm{hr}$ as measured with an electrometer from a sample exposed to $\sim 4 \mathrm{~mW}-\mathrm{cm}^{-2}$ electron power density $\left(188 \mathrm{nA}-\mathrm{cm}^{-2}\right.$ at $22 \mathrm{keV}$ ) at $135 \mathrm{~K}$. (f-g) Dependence of (f) arc rate and (g) average number of arcs per deposited charge density, as functions of beam energy for low (5 keV), intermediate (7, 10 and $15 \mathrm{keV})$, and high (22 and $25 \mathrm{keV}$ ) energy beams, with linear fits.

Even for a specific material of constant size and configuration, arc intensity variations of several orders of magnitude are not uncommon. The increased negative currents observed in most (but not all) cases were indicative of rapid dissipation of accumulated electrons from within the insulating regions of the material to the grounded sample holder. A counterexample was arcing for disordered $\mathrm{SiO}_{2}$ samples subjected to $200 \mathrm{eV}$ electron flux; at these incident energies below the second crossover energy the surface charged positively and the direction of the arcs was reversed ${ }^{3}$. Current spikes from such $\sim 1 \mu \mathrm{s}$ arcs typically have $\sim 10^{-1} \mathrm{nA}$ to $10^{2} \mathrm{nA}$ amplitude as measured with slower electrometers and $\sim 10^{-3} \mathrm{~nJ} /$ arc to $10^{3} \mathrm{~nJ} /$ arc. Arc power may be expected to scale roughly as the area of the discharge area ${ }^{24}$. Very intense arcs from large $60 \mathrm{~m}^{2}$ solar arrays have been estimated as up to $\sim 0.1 \mathrm{~J} / \mathrm{arc}^{4,22}$; scaling with area this is $\sim 200 \mathrm{~nJ} / \mathrm{arc}_{-}-\mathrm{cm}^{-2}$, a value consistent with the more intense arcs for the $1 \mathrm{~cm}$ diameter samples discussed here. Often, however, not all the 


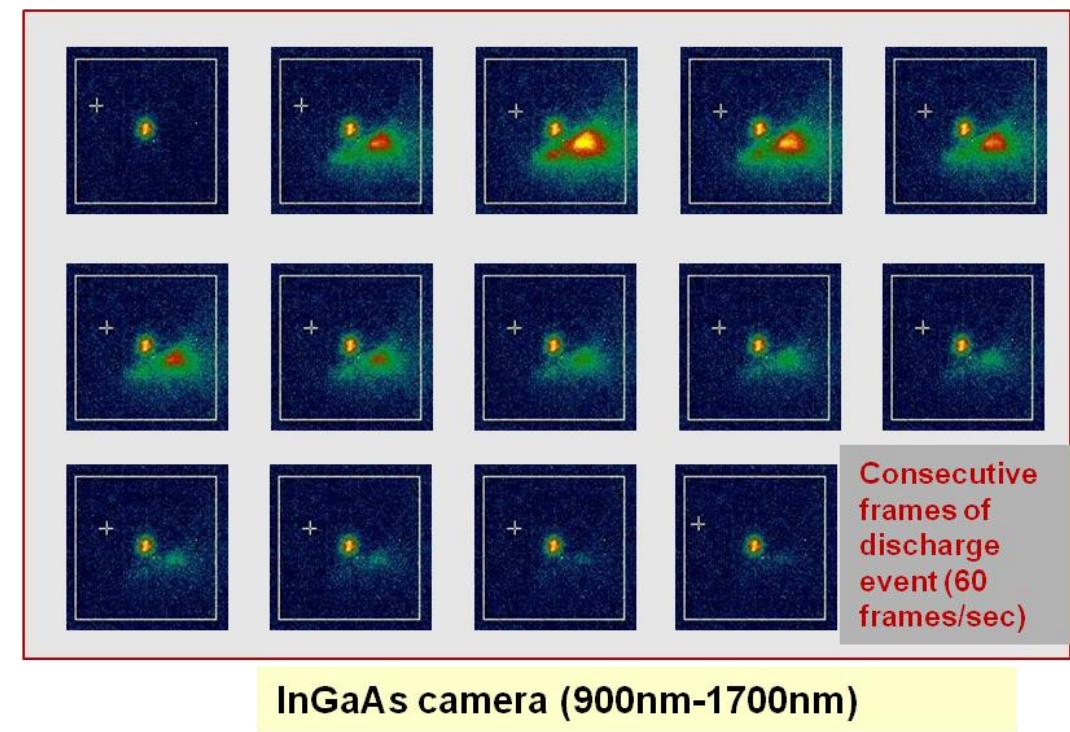

Figure 7. Typical signature of an arc event (lower right corner) for a high-conductivity carbon-loaded polyimide sample exposed to $\sim 110 \mu \mathrm{W}-\mathrm{cm}^{-2}$ electron power density $\left(5 \mathrm{nA}-\mathrm{cm}^{-2}\right.$ at $\left.22 \mathrm{keV}\right)$ at $135 \mathrm{~K}$ shown as a sequence of InGaAs video camera images at 60 frames per second. Note the small brighter spot from the blackbody radiation of the $\mathrm{LaB}_{6}$ filament of the electron gun near the center.

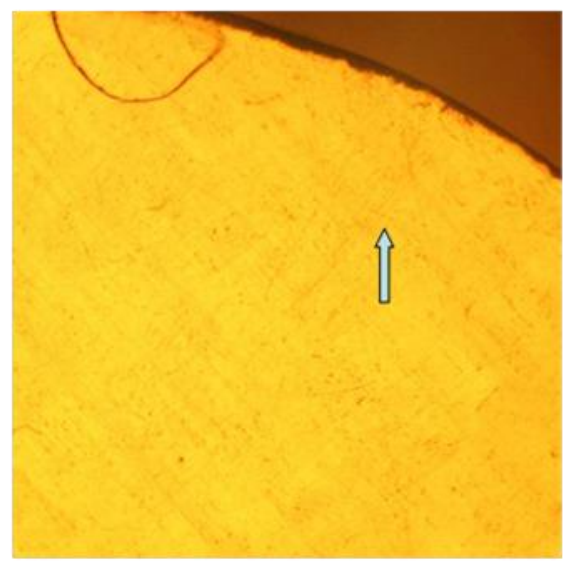

(a)

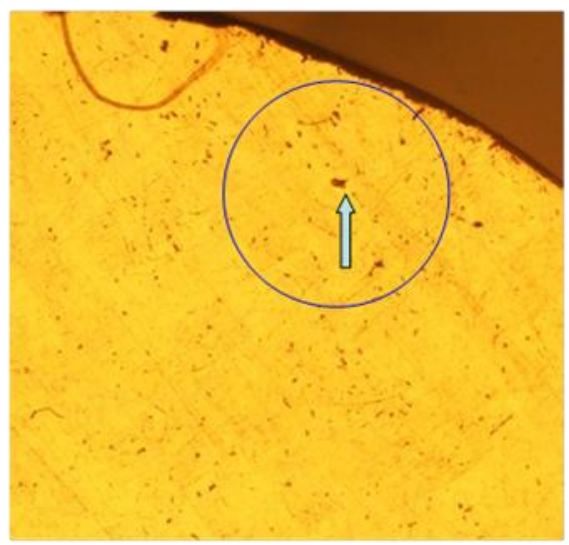

(b)

Figure 8. Comparison of optical microscope images of a composite sample surface (a) before and (b) after electron bombardment $\left(22 \mu \mathrm{W}-\mathrm{cm}^{-2}, 1 \mathrm{nA}-\mathrm{cm}^{-2}\right.$ at $\left.22 \mathrm{keV}\right)$ at $150 \mathrm{~K}$. The sample is a composite material with an ungrounded 0.1 $\mu \mathrm{m} \mathrm{Au} / \mathrm{Cr}$ coating on an epoxy resin fiberglass and carbon fiber composite substrate, with the Au side exposed to beam. Numerous damage sites from arcing are evident on the exposed sample, including the $\sim 250 \mu \mathrm{m}$ diameter feature identified with the arrow.

sample area discharges in a single event, leading to arc power that increases sub-linearly with area.

Figures 6(f) and 6(g) show the dependence for electrometer data of arc rate and average arc rate scaled by beam current density (or equivalently, number of arcs per deposited charge density) on beam energy over an incident power density range of $10^{-7} \mathrm{~W}-\mathrm{cm}^{-2}$ to $10^{-4} \mathrm{~W}-\mathrm{cm}^{-2}$. These graphs suggest that arc rate is proportional to both deposited charge density and deposited power density. The measured arcs per deposited charge density from the extreme electrometer profile in Fig. 6(e) at $\sim 4 \cdot \mathrm{mW} / \mathrm{cm}^{2}$ is approximately the same as in Figs. 6(f) and 6(g), suggesting that for this specific scenario arc rate is proportional to incident power density over 4 orders of magnitude. 


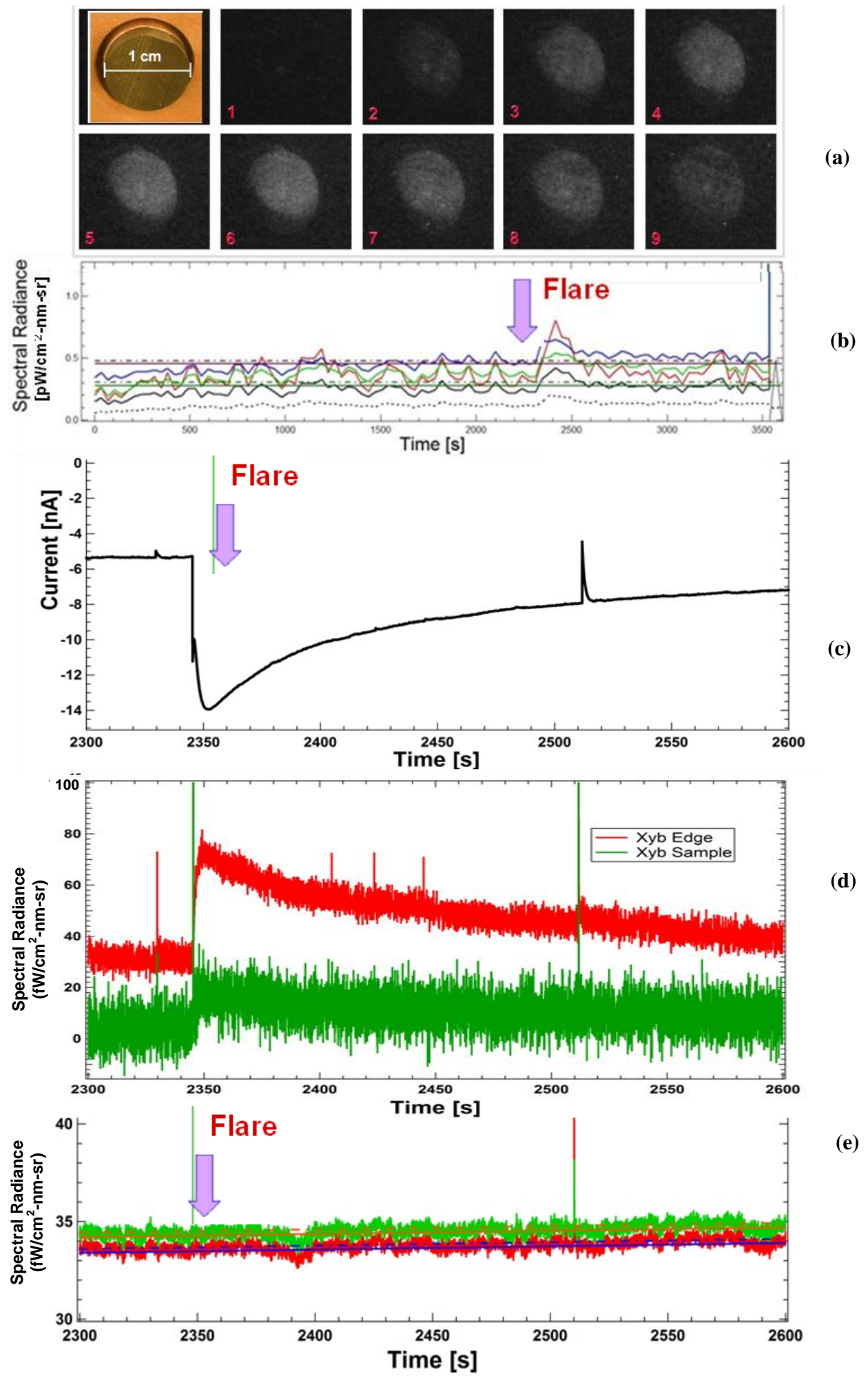

Figure 9. Typical signatures of intermediate-duration emission events for high-conductivity carbon-loaded polyimide samples exposed to $\sim 110 \mu \mathrm{W}-\mathrm{cm}^{-2}$ electron power density $\left(5 \mathrm{nA}-\mathrm{cm}^{-2}\right.$ at $\left.22 \mathrm{keV}\right)$ at $135 \mathrm{~K}$. (a) Sequence of SLR CCD camera frames at $30 \mathrm{~s} /$ frame (b) SLR CCD visible-range camera absolute spectral radiance versus time curve for this event. Intensities for the grey scale and red, green and blue components are shown as separate curves. (c) Electrometer versus elapsed time trace. Absolute spectral radiance versus time curve for (d) CCD Vis and (e) InGaAs NIR video cameras. 
In general, arc rates for a given charge deposition rate were lower for more conductive materials, which allowed greater charge dissipation. Limited measurements for three types of carbon-loaded polyimide samples with conductivities of $10^{-}$ ${ }^{3}(\Omega-\mathrm{cm})^{-1}, 10^{-5}(\Omega-\mathrm{cm})^{-1}$ and $10^{-7}(\Omega-\mathrm{cm})^{-1}$, found arc rates increased $\sim 10 \mathrm{X}$ with decreasing conductivity at comparable incident energies and current densities ${ }^{13}$. Measurements for epoxy composite materials ${ }^{14}$ and disordered $\mathrm{SiO}_{2}$ coatings ${ }^{1,13,14}$ made from < $40 \mathrm{~K}$ to $290 \mathrm{~K}$ confirmed that lower temperature samples, in general, showed larger arc rates. The dark current and radiation induced conductivities in polyimide and epoxies are several orders of magnitude lower at the lower temperatures ${ }^{23}$, leading to reduced charge dissipation and enhanced charging and electrostatic discharges at these lower temperatures.

Measurements of optical emissions from arcs have been readily observed with UV/Vis/NIR CCD, NIR InGaAs, and mid-IR InSb video cameras over wavelength ranges of $<200 \mathrm{~nm}$ to $>4 \mu \mathrm{m}^{1,12,13}$ (see Fig. 2). Arc rates and magnitudes vary greatly with material and sample geometry, however approximate values are presented here for comparison with the cathodoluminescence emissions presented above. Typical measurements of visible arc signatures from the $\sim 1 \mathrm{~cm}^{2}$ samples of carbon-loaded polyimide and disordered silica films found arcs that were $\sim 10-1000$ times the cathodoluminescent intensity with $<1 \mu$ s duration, $\sim 0.5-5 \mathrm{nA}$ amplitudes, $\sim 1 \mathrm{~nJ}$ dissipated energy, and $\sim 0.1 \operatorname{arcs}$ per J $/ \mathrm{m}^{2}$ or 10 arcs per hr from a $25 \mathrm{keV}$ electron beam at $1 \mathrm{nA} / \mathrm{cm}^{2}$. Arcs from bulk cyanate ester epoxy composite samples were 1 to 2 orders of magnitude larger, at about 1 order of magnitude higher arc rate.

As might be expected for short-duration arcs, the optical emissions cover a very wide range of frequencies. High frequency arc signatures from arcs on solar array samples, in radio frequencies from $20 \mathrm{kHz}$ to $1 \mathrm{GHz}(0.3 \mathrm{~m}$ to $20 \mathrm{~km})$, have also been observed with antennas near carbon-loaded polyimide samples exposed to an electron beam ${ }^{22,25}$. Leung measured arcs on solar array samples with rise times of $\sim 20 \mathrm{~ns}$ and the fall times of $\sim 300 \mathrm{~ns}$; corresponding roughly to frequencies $\sim 5 \mathrm{MHz}$ to $50 \mathrm{MHz}$ with frequency content at $>50 \mathrm{MHz}$ in higher order harmonics ${ }^{22}$. Although the radio frequency emission falls off rapidly with decreasing wavelength (The electric field strength falls off by about $75 \mathrm{~dB}$ from $1 \mathrm{MHz}$ to $1 \mathrm{GHz}$.), these emissions can be intense as compared with background emissions ${ }^{4}$. The radio emissions from large arcs are much brighter than the reflected solar radio emissions, even in direct sunlight. Even at $1 \mathrm{GHz}$, the emitted radio frequency fluxes are similar to those seen in solar radio outbursts ${ }^{4}$.

\subsection{Intermediate-duration Charging/Discharging}

Intermediate-duration emissions termed "flares" have occasionally been observed in polymeric dielectric materials under prolonged electron bombardment. Flares have been seen in polyimide films, neat urethane and bisphenol/amine epoxy films, carbon-loaded polyimide ${ }^{13,14}$, and cyanate ester and urethane epoxy resin composites ${ }^{13}$, as shown in Fig. 2. Flares have not been observed in disordered $\mathrm{SiO}_{2}$ coatings ${ }^{1}$. The features are seen simultaneously in current and Vis and NIR spectral response curves (see Figs. 4 and 9). Observations with cameras equipped with filters showed that most of the emission from flares is in $\sim 300 \mathrm{~nm}$ to $\sim 1000 \mathrm{~nm}$ wavelength range. The optical emissions tend to emanate from the full charged surface, suggesting they are related to charging and dissipation over large areas. Flares are infrequent (typically 1 to 2 flares/hr) and have only observed in long runs after $\gtrsim 20$ min beam exposure, which suggests the necessity for substantial charging within the sample before flares can occur.

Flares have abrupt onset rise times $(<0.1 \mathrm{~s})$, believed to be associate with a rapid discharge. Flares (usually) have an arc event associated with their instigation, although the origin of such large arc triggers is not known. Flares also exhibit very long times $\left(\sim 10^{1}-10^{2} \mathrm{~s}\right)$ for the currents or spectral radiance to return to pre-flare equilibrium values associated with sustained glow. The response for individual flares between the abrupt onset and long-term decay is complex and can vary from one flare to the next.

Too few flares have been observed to accurately determine the flare dependence on current density, charge fluence, beam energy, or deposited power. Flares do seem to occur more frequently for higher energies. Roughly speaking, the spectral response and electrometer currents $(\sim 1-100 \mathrm{nA}$ amplitude with $<1-10 \mu \mathrm{J})$ of flares are $\sim 2-20 \mathrm{X}$ that observed for typical sustained glow; power from flares is typically $\sim 5 \%$ to $20 \%$ of cathodoluminescent power.

\section{CONCLUSION}

Three types of electron-induced optical emissions were observed in a variety of dielectric spacecraft materials:

(i) long-duration cathodoluminescence, which persists as long as the electron beam is on;

(ii) short-duration (<1 s) arcing, resulting from electrostatic discharge; and

(iii) intermediate-duration ( 100 s) glow-termed "flares". 
We described how the electron currents and arcing — as well as light emission absolute intensity and frequency—depend on incident electron beam energy, flux, and power, and on material composition, temperature and thickness for different bulk and composite insulating materials. A general theory for emission cathodoluminescent intensity, based on the disordered band structure of the materials, has been proposed that describes the basic observations for these systems ${ }^{1,12-}$ 14. Because the arcing rate and discharge magnitude depend so much on the specific geometries and conditions, it is difficult to estimate the arc emission intensity without custom testing for specific missions and conditions ${ }^{24}$. Ferguson et al. discusses the remote observation of these optical emissions, with comparisons of their intensities to background light in the LEO and GEO space environments ${ }^{4}$.

Based on the studies described in this paper, we conclude that the designers of space-based observatories should consider space environment electron-induced optical emissions as potential local sources of stray light. However, a number of factors will determine how significant these contributions will be, and these factors will generally be mission specific. To determine the magnitude of electron-induced optical emissions from observatory components for a specific space-based observatory situation requires knowledge of ${ }^{1}$ :

(i) The observatory electron environment, and specifically the electron differential electron flux spectra;

(ii) The differential electron flux spectra reaching specific optical component that may cathodoluminesce or arc;

(iii) The effectiveness of the optical system in capturing the optical emissions, including the juxtaposition of the luminescent elements to the optical path;

(iv) The specific thickness of the material, particularly as related to the energy-dependant penetration range of incident electrons;

(v) The temperature of the material;

(vi) The specific composition of the material, as well as surface morphology and contamination; and

(vii) The charge storage and transport properties of the material, including changes of conductivity with electric field, temperature, and dose rate.

Numerous factors have been determined which enhance the impact of these emissions and should also be taken into consideration in future observatory designs. These include:

(i) high flux and high variability environments;

(ii) high sensitivity imaging; complex, sensitive optical systems and electronics;

(iii) low temperature operations which reduce thermal emissive background from observatory components and decrease conductivity thereby enhancing charge accumulation and discharge;

(iv) large areas of dielectric materials that can produce optical emissions;

(v) open architectures and minimal shield that expose materials to greater electron fluxes;

(vi) long-duration missions that permit extended charge accumulation; and

(vii) remote and long-duration missions that preclude system maintenance.

\section{ACKNOWLEDGMENTS}

We gratefully acknowledge contributions to instrumentation and experimental efforts from Robert Johnson, Jennifer Albretsen Roth and Doug Ball of the USU Materials Physics Group, help with optical calibrations from James Peterson of the USU Space Dynamics Laboratory, and Michael Taylor for the use of infrared and CCD video cameras. We also acknowledge the many useful discussions about this work from contributors to the JWST Electrical Systems Working Group conference calls, especially James Heaney and Todd Schneider. This work was supported by projects through the NASA Goddard Space Flight Center. Graduate and undergraduate student research support was also provided by a NASA Space Technology Research Fellowship (Jensen), and the Utah State University Office of Research and Graduate Studies (Ball, Dekany, Jensen, Johnson, Roth, Wilson) and the College of Science (Jensen, Roth). 


\section{REFERENCES}

[1] Jensen, A. E., Dennison, J. R., Wilson, G., Dekany, J., Bowers, C.W., Meloy, R. and Heaney, J. B., "Consequences of Cathodoluminescence for Cryogenic Applications of $\mathrm{SiO}_{2}$-based Space Observatory Optics and Coatings," Proc. SPIE Cryogenic Optical Systems and Instrum. Conf., Paper No. 8863-11, San Diego, CA, (2013).

[2] Lightsey, P. A., Atkinson, C., Clampin, M., and Feinberg, L. D., "James Webb Space Telescope: large deployable cryogenic telescope in space", Opt. Eng. 51(1), 011003 (2012).

[3] Wilson, G., Evans, A., Dekany, J. and Dennison, J. R., "Charging Effects of Multilayered Dielectric Spacecraft Materials: Surface Voltage, Discharge and Arcing,” IEEE Trans. on Plasma Sci., (2013), in press.

[4] Ferguson, D. C., Krezan, J. -M., Barton, D. A., Dennison, J. R. and Gregory, S., "On the Feasibility of Detecting Spacecraft Charging and Arcing by Remote Sensing," Paper Number, AIAA-2013-2828, $5^{\text {th }}$ AIAA Atmospheric and Space Environments Conference, San Diego, CA, (2013).

[5] Wei, Z. and Lightsey, P.A., "Stray light from galactic sky and zodiacal light for JWST", Proc. SPIE 6265, Space Telescopes and Instrumentation I: Optical, Infrared, and Millimeter, 62653C, June (2006).

[6] Leinert, C. et al., "The 1997 Reference of Diffuse Night Sky Brightness," Astron. Astrophys. Suppl. Ser., 127, 1.

[7] Lightsey, P.A., and Wei, Z., "James Webb Space Telescope observatory stray light performance", Proc. SPIE6265, Space Telescopes and Instrumentation I: Optical, Infrared, and Millimeter, 62650S, June (2006).

[8] Hastings, D. and Garrett H., [Spacecraft-Environment Interactions], New York, NY: Cambridge Press, (1996).

[9] Thomson, C.D., Zavyalov, V., and Dennison, J.R. "Instrumentation for Studies of Electron Emission and Charging from Insulators," Proc. $8^{\text {th }}$ Spacecraft Charging Techn. Conf. (NASA Marshall Space Flight Center, Huntsville, Al, (2003).

[10] Dennison, J. R., "Characterization of Electrical Materials Properties Related to Spacecraft Charging," Radiation Capabilities for the Europa Jupiter System Missions Instrument Workshop, Johns Hopkins Applied Physics Laboratory, Laurel, MD, July (2009).

[11] Dekany, J., Johnson, R. H., Wilson, G., Evans, A. and Dennison, J. R., "Ultrahigh Vacuum Cryostat System for Extended Low Temperature Space Environment Testing," IEEE Trans. on Plasma Sci., (2013), in press.

[12] Dennison, J. R., Evans, A., Wilson, G., Dekany, J., Bowers C. W., and Meloy, R., "Electron Beam Induced Luminescence of $\mathrm{SiO}_{2}$ Optical Coatings," Proc. IEEE-CEIDP (2012).

[13] Jensen, A. E., Dennison, J. R., Wilson, G. and Dekany, J., "Nanodielectric Properties of High Conductivity CarbonLoaded Polyimide under Electron-Beam Irradiation," Proc. ICSD, (2013).

[14] Jensen, A. E., Wilson, G., Dekany, J., Sim, A. M. and Dennison, J. R, "Low Temperature Cathodoluminescence of Space Observatory Materials," IEEE Trans. on Plasma Sci., (2013), in press.

[15] Griseri, V., Dissado, L. A., Fothergill, J. C., Laurent, C. and Teyssedre, G., "Photoluminescence, recombination induced luminescence and electroluminescence in epoxy resin,” J. Phys. D: Appl. Phys, 34, 2534-2540 (2001).

[16] Fitting, H. -J., Barfels, T., von Czarnowski, A. and Trukhin, A. N., "Electron Beam Induced Optical and Electronical Properties of $\mathrm{SiO}_{2}$," Materials Science and Engineering, B71, 109-114 (2000).

[17] Fitting, H. -J., Barfels, T., Trukhin, A. N. and Schmidt, B., "Cathodoluminescence of crystalline and amorphous $\mathrm{SiO}_{2}$ and $\mathrm{GeO}_{2}$," J. Non-Crystalline Solids, 279, 51-59 (2000).

[18] Salh, R., von Czarnowski, A., Zamoryanskaya, M. V., Kolesnikova E. V. and Fitting, H. -J., "Cathodoluminescence of $\mathrm{SiO}_{\mathrm{x}}$ Under-stoichiometric Silica Layers,” Phys. Stat. Sol., 203, 2049-2057 (2006).

[19] Trukhin, A. N., Goldberg, M., Jansons, J., Fitting, H. -J. and Tale, I. A., "Silicon Dioxide Thin Film Luminescence in Comparison with Bulk Silica," J. Non-Crystalline Solids, 223, 114-122 (1998).

[20] Wilson, G. and Dennison, J. R., "Approximation of Range in Materials as a Function of Incident Electron Energy," IEEE Trans. on Plasma Sci., 40(2), 305-310 (2012).

[21] Dennison, J. R., Wilson, G., Hoffmann, R. and Evans, A., "Comparison of Radiation Induced Conductivities at Low Temperature,” Bull. Am. Phys. Soc. 57(1), APS March Meeting, Baltimore, MD, March (2013).

[22] Leung, P., "Characterization of EMI generated by the discharge of VOLT solar array," NASA-CR-176537, (1985).

[23] Roth, J.A., Hoffmann, R. and Dennison, J.R., "Effects of radiation induced conductivity on electrostatic discharge in insulating materials," Proc. $1^{\text {st }}$ AIAA Atmospheric and Space Environments Conf., paper no. AIAA-2009-3527, San Antonio, TX, (2009).

[24] Frederickson, A. R., "New Scaling Laws for Spacecraft Discharge Pulses," Proc. $7^{\text {th }}$ Spacecraft Charging Tech. Conf., Noordwijk, Netherlands, (2001).

[25] Wallach, E., NASA Goddard Space Flight Center, unpublished, (2012). 